\section{Collagen solubility correlates with skin optical clearing}

\author{
Jason Hirshburg, ${ }^{a}$ Bernard Choi, ${ }^{b}$ J. Stuart Nelson, ${ }^{b}$ and \\ Alvin T. Yeh ${ }^{\mathrm{a}, *}$ \\ ${ }^{a}$ Texas A\&M University \\ Department of Biomedical Engineering \\ 337 Zachry Engineering Center, 3120 TAMU \\ College Station, Texas 77843 \\ beckman Laser Institute and Medical Clinic \\ University of California \\ Irvine, California 92612
}

Abstract. Biomedical optics and photomedicine applications are challenged by the turbidity of most biological tissue systems. Nonreactive, biocompatible chemical agents can induce a reversible reduction in optical scattering of collagenous tissues such as human skin. Herein we show that a chemical agent's tissue optical clearing potential is directly related to its collagen solubility, providing a rational design basis for effective, percutaneous formulations. ๑ 2006 Society of Photo-Optical Instrumentation Engineers. [DOI: 10.1117/1.2220527]

Keywords: tissues; optical properties; collagen; lasers.

Paper 06087LR received Mar. 31, 2006; revised manuscript received May 23, 2006; accepted for publication May 25, 2006; published online Jul. 11, 2006.

Success of light-based therapies depends on sufficient photon energy reaching subsurface targets. This can be problematic since most biological tissues are highly light scattering, dispersing much of the incident photon energy from intended targets, thereby reducing therapeutic efficacy. For dermatologic applications, the optical properties of human skin, ${ }^{1}$ in particular light scattering by ubiquitous, high-order collagen structures within the dermis, ${ }^{2}$ prevent delivery of collimated light to the desired targets. These same properties prove to be formidable barriers to the collection of light (or optical signal) from within as in the case of diagnostic fluorescence or whole-body bioluminescence studies. As a consequence, many optical devices have been designed for use in the nearinfrared spectral region where tissue light scattering and absorption are minimal.

Traditionally, tissue optical properties have been considered fixed. However, after tissue immersion in particular nonreactive chemical agents, a temporary reduction in light scattering has been demonstrated. ${ }^{3}$ Optical clearing can be dramatic as tissue becomes visibly transparent and loses mechanical compliance. Upon immersion in physiological saline, tissue turbidity and mechanical properties return to their native states. ${ }^{4}$ Common properties of these chemical agents such as sugars and sugar-alcohols have suggested refractive index matching with native tissue components, in particular collagen, and dehydration are possible mechanisms of tissue optical clearing. ${ }^{3}$ However, a mechanistic understanding of tis-

*Tel: 979-845-5468; E-mail: ayeh@tamu.edu sue optical clearing using refractive index matching remains to be substantiated.

Previous studies suggest a consequence and necessary condition of tissue optical clearing is the destabilization of highorder collagen structures; after tissue immersion in compounds used for fixation, optical clearing does not occur. ${ }^{5}$ In fact, many of the chemical agents used for optical clearing also have been used in collagen self-assembly studies to characterize and understand the dynamics of fibrillogenesis. ${ }^{6}$ Herein we use solubility tests to characterize chemical agentcollagen interaction for a series of sugar-alcohols (ethylene glycol, glycerol, xylitol, and sorbitol) and propanediols (1,2propanediol and 1,3-propanediol) and correlate this data with optical clearing potential measurements in rodent and human skin in vitro.

We first characterized the inhibitory effects of chemical agents on collagen self-assembly. In vitro self-assembly of solubilized $(\mathrm{pH} \sim 3)$ rodent tail collagen I (BD Biosciences) into fibrils was carried out in phosphate buffered saline (PBS) $(0.138 \mathrm{M} \mathrm{NaCl}, 0.0027 \mathrm{M} \mathrm{KCl})$. With addition of $\mathrm{NaOH}$, collagen solutions were adjusted to physiological $\mathrm{pH}(\sim 7.4)$, inducing collagen fibrillogenesis, and incubated at $37^{\circ} \mathrm{C}$ for $24 \mathrm{~h}$. After fibrillogenesis was complete, solutions were centrifuged at $12,000 \mathrm{rpm}$ for $15 \mathrm{~min}$ to separate fibrils (white precipitate) from the remaining collagen molecules (supernatant). Collagen fibril formation was estimated by UV $(276 \mathrm{~nm})$ absorbance of supernatant before and after solution neutralization. Preliminary measurements found 32\% of collagen was assembly incompetent. Fibril formation and collagen solubility are reported relative to the assembly competent collagen content. Estimated fibril formation is shown in Fig. 1(a) for a 1.4 M chemical agent concentration. Straight lines are guides to the eye and calculated by linear regression. Ethylene glycol and 1,2-propanediol showed negligible inhibitory effects on collagen fibrillogenesis. With these agents, efficiency of fibril formation was near unity, mirroring collagen self-assembly in PBS (data not shown). Longer-chain polyols such as glycerol, xylitol, sorbitol, and 1,3-propanediol showed increasing inhibition of collagen fibrillogenesis with increasing chemical agent concentration, consistent with previously reported results. ${ }^{7,8}$

Collagen solubility as a function of chemical agent concentration is shown in Fig. 1(b). Solubility is quantified as the amount of collagen remaining in supernatant following fibrillogenesis. In this data set, the initial collagen concentration was $1.25 \mathrm{mg} / \mathrm{ml}$. As would be expected from the data shown in Fig. 1(a), ethylene glycol and 1,2-propanediol showed low collagen solubilities. With glycerol, 1,3-propanediol, xylitol, and sorbitol solutions, their collagen solubilities increased with increasing chemical agent concentration. Sorbitol exhibited twice the collagen solubility of glycerol and 1,3propanediol, consistent with previously reported results. ${ }^{8}$ Overall, our data demonstrated an increase in collagen solubility with increasing sugar-alcohol chain length from ethylene glycol (two carbon chain) to sorbitol (six carbon chain).

Chemical agents that can induce tissue optical clearing increase collagen solubility by suppressing hydrophilic, intermolecular interactions. ${ }^{6}$ The propanediols evaluated in this study have been used previously to highlight the role of hy-

1083-3668/2006/11(4)/040501/3/\$22.00 @ 2006 SPIE 


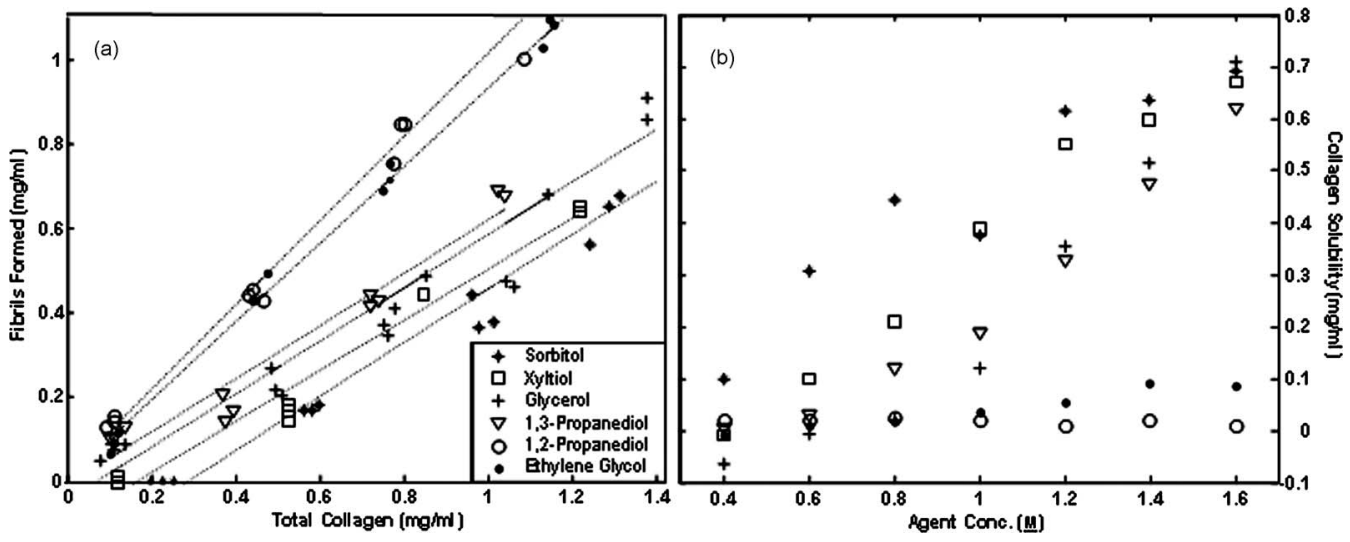

Fig. 1 Collagen fibril formation and solubility. (a) Fibrils formed from total collagen in 1.4 M solutions of each chemical agent. (b) Quantity of collagen remaining in solution (initial concentration of $1.25 \mathrm{mg} / \mathrm{ml}$ ) as a function of chemical agent concentration.

drogen bonding in collagen fibrillogenesis. ${ }^{8}$ Suppression of these hydrophilic attractive forces can destabilize high-order collagen structures in native and in vitro tissues as observed microscopically ${ }^{5}$ and ultrastructurally. ${ }^{9}$ Having characterized chemical agent collagen solubilities, we then investigated whether the ability of each agent to suppress hydrogen-bondmediated attractive forces was correlated with its optical clearing potential (OCP) on in vitro skin.

Using an integrating-sphere-based method, ${ }^{10} \mathrm{OCP}$ of each chemical agent was evaluated in rodent (from 3- to 6-weekold animals) and human skin. Harvested rodent skin was stored in PBS at $4{ }^{\circ} \mathrm{C}$ until experiments were performed less than $24 \mathrm{~h}$ later. Rodent and cryopreserved $\left(-20^{\circ} \mathrm{C}\right)$, dermatomed human skin were cut into $1.5 \times 1.5 \mathrm{~cm}^{2}$ samples using surgical scissors. Subcutaneous fat was removed using a razorblade and skin thickness was measured using a micrometer (Mitutoyo) after the sample had been placed between two glass slides of known thickness. Transmitted and reflected 635-nm laser light and skin thickness were measured before and after application of specific chemical agents to the dermal side of the skin samples; experiments were performed at room temperature. Chemical agent solutions were volume matched to skin samples; skin samples were exposed for $45 \mathrm{~min}$ to chemical agent solutions. The inverse-adding doubling method $^{11}$ was used to calculate the reduced scattering coefficient $\mu_{s}^{\prime}$ before and after chemical agent application. Data are reported as the reduced scattering ratio, RSR $=\mu_{s}^{\prime}($ before $) / \mu_{s}^{\prime}($ after $)$. Each data point is an average of at least four measurements.

RSR as a function of chemical agent concentration is shown for rodent skin in Fig. 2(a). The slope from linear regression analysis of RSR data was used to define OCP for each chemical agent. Application of one-way analysis of co-

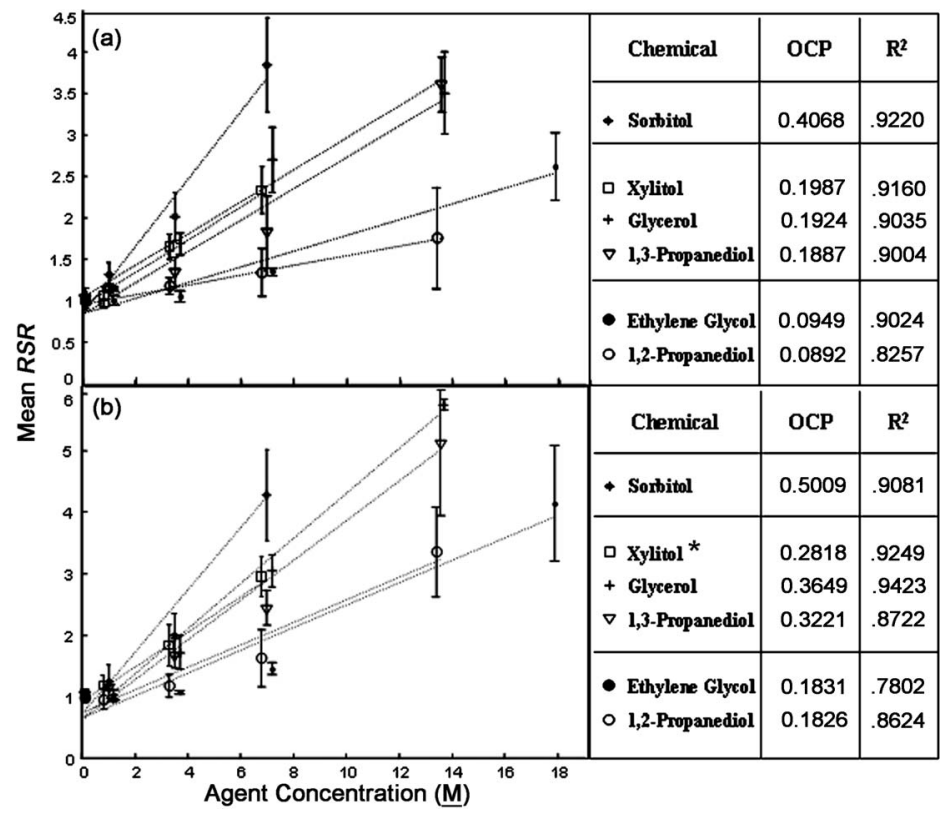

Fig. 2 Reduced scattering ratio (RSR) of chemical agents in rodent (a) and human (b) skin. Slope of linear regression analysis of RSR yields optical clearing potential (OCP) of each chemical agent, shown at right. *Xylitol OCP was not significantly different from that of glycerol, 1,3-propanediol, ethylene glycol, or 1,2-propanediol. 
Table 1 Chemical agent osmolality $(\mathrm{Osm} / \mathrm{kg})$, refractive index $\left(n_{D^{\prime}}\right.$ $20^{\circ} \mathrm{C}$ ), and molecular weight.

\begin{tabular}{lccc}
\hline Chemical & $\begin{array}{c}\text { Osmolality } \\
(\text { Osm/kg) }\end{array}$ & $\begin{array}{c}\text { Refractive } \\
\text { Index }\end{array}$ & $\begin{array}{c}\text { Molecular } \\
\text { Weight }\end{array}$ \\
\hline Sorbitol & 15.2 & 1.45 & 182.17 \\
Xylitol & 15.6 & 1.45 & 152.15 \\
Glycerol & 7.6 & 1.46 & 92.09 \\
1,3-Propanediol & 8.3 & 1.44 & 76.10 \\
1,2-Propanediol & 8.7 & 1.43 & 76.10 \\
Ethylene Glycol & 9.0 & 1.43 & 62.07 \\
\hline
\end{tabular}

variance test to the RSR data identified three significantly different groups $(p \leq 0.005)$ : sorbitol; xylitol, glycerol, and 1,3-propanediol; and ethylene glycol and 1,2-propanediol. Of the agents tested, sorbitol had the highest OCP, twice that for xylitol, glycerol, and 1,3-propanediol. In turn, xylitol, glycerol and 1,3-propanediol had twice the OCP of ethylene glycol and 1,2-propanediol.

The RSR of each agent showed similar trends in human skin [Fig. 2(b)]. OCP values were slightly greater in human skin as compared to rodent, indicative of a greater optical clearing effect. Three groups were found to be significantly different $(p \leq 0.05)$ : sorbitol; glycerol and 1,3-propanediol; and ethylene glycol and 1,2-propanediol. The RSR data of xylitol were not significantly different from ethylene glycol, glycerol, 1,2- or 1,3-propanediol. Sorbitol had the highest OCP in human skin. Glycerol and 1,3-propanediol had twice the OCP of ethylene glycol and 1,2-propanediol.

Our data indicate that a chemical agent's ability to suppress hydrogen-bond-mediated attractive forces within collagen directly correlates with its OCP. Sorbitol had twice the collagen solubility of glycerol and, correspondingly, exhibited twice the OCP. Traditionally, refractive index matching with collagen $(n=1.45-1.55)^{2,12}$ had been used empirically to screen and select potential chemical agents for tissue optical clearing. ${ }^{3,13}$ The propanediols used in our study had similar physical properties including molecular weight, index of refraction and osmolality (see Table 1), but the most important characteristic predictive of their OCP was collagen solubility. Similar comparisons can be made with the sugar-alcohol series; no correlation can be made between the chemical agent's index of refraction or osmolality with its OCP. For sugaralcohols, our study suggests that an even higher OCP may be realized by extending chain length. Other chemical agents with higher collagen solubilities than sorbitol, namely glucose and fructose, ${ }^{8}$ may correspondingly exhibit higher OCP. In fact, a reduction in tissue $\mu_{s}^{\prime}$ has been correlated with increased glucose concentration in studies to develop noninvasive optical monitoring methods. ${ }^{10,14}$

Many of the effective optical clearing chemical agents presented herein are already used as sweetening additives in foods and emollients in skin care products. A remaining challenge for their use in conjunction with light-based dermatologic therapeutics (and diagnostics) is percutaneous delivery through the intact stratum corneum of human skin. Some success has been reported when chemical agents were chaperoned with lipophilic compounds. ${ }^{15}$ We believe that the results reported herein provide a foundation for rational design and development of effective, topically-applied, optical clearing formulations.

\section{Acknowledgments}

The authors thank Mr. Justin Lotfi at Beckman Laser Institute for assistance with preliminary RSR data acquisition and Dr. Jerry McCullough at University of California, Irvine, for his assistance in obtaining human skin samples. This work was funded by National Science Foundation Faculty Early Career Development (CAREER) Award (A.T.Y.), Beckman Fellows Program (B.C.), the National Center for Research Resources at the National Institutes of Health (Laser Microbeam and Medical Program, RR01192), and National Institutes of Health (EB004846 to A.T.Y. and AR047551, EB002495, and AR048458, to J.S.N.).

\section{References}

1. R. R. Anderson and J. A. Parrish, "The optics of human skin," J. Invest. Dermatol. 77(1), 13-19 (1981).

2. I. S. Saidi, S. L. Jacques, and F. K. Tittel, "Mie and Rayleigh modeling of visible-light scattering in neonatal skin," Appl. Opt. 34(31), $7410-7418$ (1995)

3. V. V. Tuchin, Optical Clearing of Tissues and Blood, SPIE Press, Bellingham, WA (2005) and references therein.

4. P. B. Wells, A. T. Yeh, and J. D. Humphrey, "Influence of glycerol on the mechanical behavior and thermal damage susceptibility of collagenous tissues," IEEE Trans. Biomed. Eng. 53(4), 747-753 (2006).

5. A. T. Yeh, B. Choi, J. S. Nelson, and B. J. Tromberg, "Reversible dissociation of collagen in tissues," J. Invest. Dermatol. 121(6), 1332-1335 (2003).

6. A. T. Yeh and J. Hirshburg, "Molecular interactions of exogenous chemical agents with collagen-implications for tissue optical clearing," J. Biomed. Opt. 11(1), 014003 (2006) and references therein.

7. G. C. Na, L. J. Butz, D. G. Bailey, and R. J. Carroll, "In vitro collagen fibril assembly in glycerol solution: evidence for a helical cooperative mechanism involving microfibrils," Biochemistry 25, 958 966 (1986).

8. N. Kuznetsova, S. L. Chi, and S. Leikin, "Sugars and polyols inhibit fibrillogenesis of type I collagen by disrupting hydrogen-bonded water bridges between the helices," Biochemistry 37, 11,888-11,895 (1998).

9. L. Leonardi, A. Ruggeri, N. Roveri, A. Bigi, and E. Reale, "Light microscopy, electron microscopy, and x-ray diffraction analysis of glycerinated collagen fibers," J. Ultrastruct. Res. 85, 228-237 (1983).

10. B. Choi, L. Tsu, E. Chen, T. S. Ishak, S. M. Iskandar, S. Chess, and J. S. Nelson, "Determination of chemical agent optical clearing potential using in vitro human skin," Lasers Surg. Med. 36, 72-75 (2005).

11. S. A. Prahl, M. J. C. van Gemert, and A. J. Welch, "Determining the optical properties of turbid media by using the adding-doubling method," Appl. Opt. 32, 559-568 (1993).

12. M. Gisselberg, J. I. Clark, S. Vaezy, and T. B. Osgood, "A quantitative evaluation of Fourier components in transparent and opaque calf cornea," Am. J. Anat. 191, 408-418 (1991).

13. G. Vargas, E. K. Chan, J. K. Barton, H. G. Rylander III, and A. J. Welch, "Use of an agent to reduce scattering in skin," Lasers Surg. Med. 24, 133-141 (1999).

14. J. T. Bruulsema et al., "Correlation between blood glucose concentration in diabetics and noninvasively measured tissue optical scattering coefficient," Opt. Lett. 22(3), 190-192 (1997).

15. M. H. Khan, B. Choi, S. Chess, K. M. Kelly, J. McCullough, and J. S. Nelson, "Optical clearing of in vivo human skin: implications for light-based diagnostic imaging and therapeutics," Lasers Surg. Med. 34, 83-85 (2004). 